\title{
Las luces del terremoto de Guayaquil del 16 de abril de 2016: Un estudio de la correlación con la aceleración
}

\author{
Juan Antonio Lira Cacho \\ Facultad de Ciencias Físicas, Universidad Nacional Mayor de San Marcos, Ciudad Universitaria, Lima, Perú \\ Sección Física, Pontificia Universidad Católica del Perú, Ciudad Universitaria, Lima, Perú \\ Enviado el 15 de junio del 2021. Aprobado el 29 de junio del 2021.
}

DOI: https://doi.org/10.33017/RevECIPeru2021.0003/

\section{Resumen}

En los últimos años, las evidencias sobre las luces sísmicas (EQL) se han fortalecido con grabaciones de películas. Este es el caso de los fenómenos luminosos que se han observado en Guayaquil durante el terremoto del 16 de abril de 2016. Las inusuales luces fueron captadas por una cámara de seguridad instalada en el campus de la Escuela Superior Politécnica del Litoral (ESPOL) (Fig. 1). Hasta hace unas décadas, los EQL eran tomados con cierto escepticismo. La falta de datos concretos dificultaba su aceptación. La grabación de la película sobre los EQL de Guayaquil es a la vez impresionante y útil para el estudio de los EQL. En este trabajo se encontró una correlación temporal diferencial entre los EQL y las aceleraciones sísmicas del suelo. Una mejor comprensión del proceso de generación de los EQL puede mejorar nuestra comprensión de la sismicidad y ayudar en la predicción de terremotos.

Descriptores: ondas sísmicas, fracturación de roca, luces sísmicas, correlación temporal diferencial, electromagnetismo

\begin{abstract}
In recent years, the evidences about the earthquake lights (EQL) have strengthened with film recordings. This is the case of the light phenomena which have been observed in Guayaquil during the earthquake of 16 April 2016. The unusual lights were captured by a security camera installed at the Escuela Superior Politécnica del Litoral (ESPOL) campus (Fig. 1). Up until a few decades ago, EQL were taken with some skepticism. The lack of hard data made it difficult to accept them. The film recording about the Guayaquil EQL is both impressive and useful for the study of the EQL. In this work a time difference correlation between the EQL and the seismic ground accelerations was found. A better understanding of the EQL generation process can improve our understanding of seismicity and help in the prediction of earthquakes.
\end{abstract}

Keywords: seismic waves, rock fracturing, seismic lights, time difference correlation, electromagnetism. 


\section{Introducción}

El terremoto de Ecuador, del 16 de abril de 2016, se originó en el proceso de convergencia entre las placas de Nazca y Sudamericana [1]. La ubicación del epicentro y las isosísmicas de la Escala Macrosísmica Europea 1998 (EMS-98) se muestran en la Fig. 2. Las aceleraciones sísmicas del suelo se registraron en la estación sismológica AMIL ubicada a $39 \mathrm{~km}$ de Guayaquil. En este estudio se utilizaron los datos sísmicos registrados en esta estación. Los registros de aceleración del terremoto se obtuvieron del IGEPN y se pueden descargar gratuitamente para uso científico. La presentación gráfica de los datos se muestra en la Fig. 3. La grabación de la película que muestra los EQL de Guayaquil es de libre acceso en la web https://www.youtube.com/watch?v=Ddt2BV0CS68. Maurizio Mulas, de ESPOL, confirmó, que los EQL se observaron en Guayaquil durante el choque sísmico principal. Además, en el mapa que se muestra en la Fig. 4 marcó con el círculo rojo la ubicación de la cámara y con las dos líneas rojas, aproximadamente, el campo de visión de la cámara. También señaló con la línea discontinua azul la falla Chongón-Colonche que pasa por la región. Según Mulas, ésta podría ser la zona donde se generaron los EQL. La película tiene una duración de 56 segundos y contiene una grabación continua del evento, proporcionada por una cámara giratoria a color. Se cronometraron quince EQL de la grabación de la película y luego para el primer EQL se asignó el origen del tiempo relativo (0 s). Esto significa que solo se consideraron las diferencias de tiempo entre el primer EQL y los siguientes. Las diferencias de tiempo de los EQL se muestran en la Fig.5.

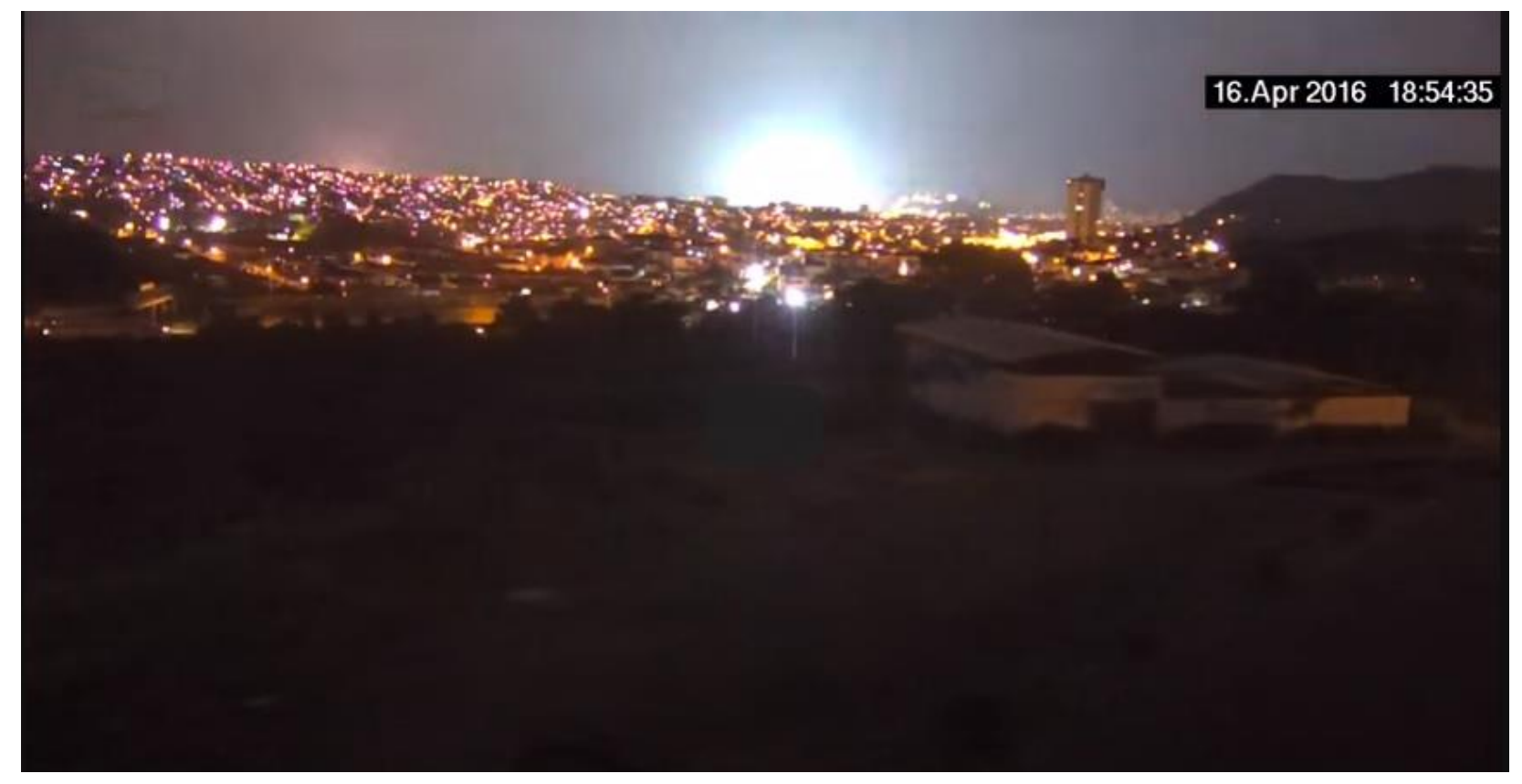

Figura 1: Luz sísmica de Guayaquil grabada en la película. 


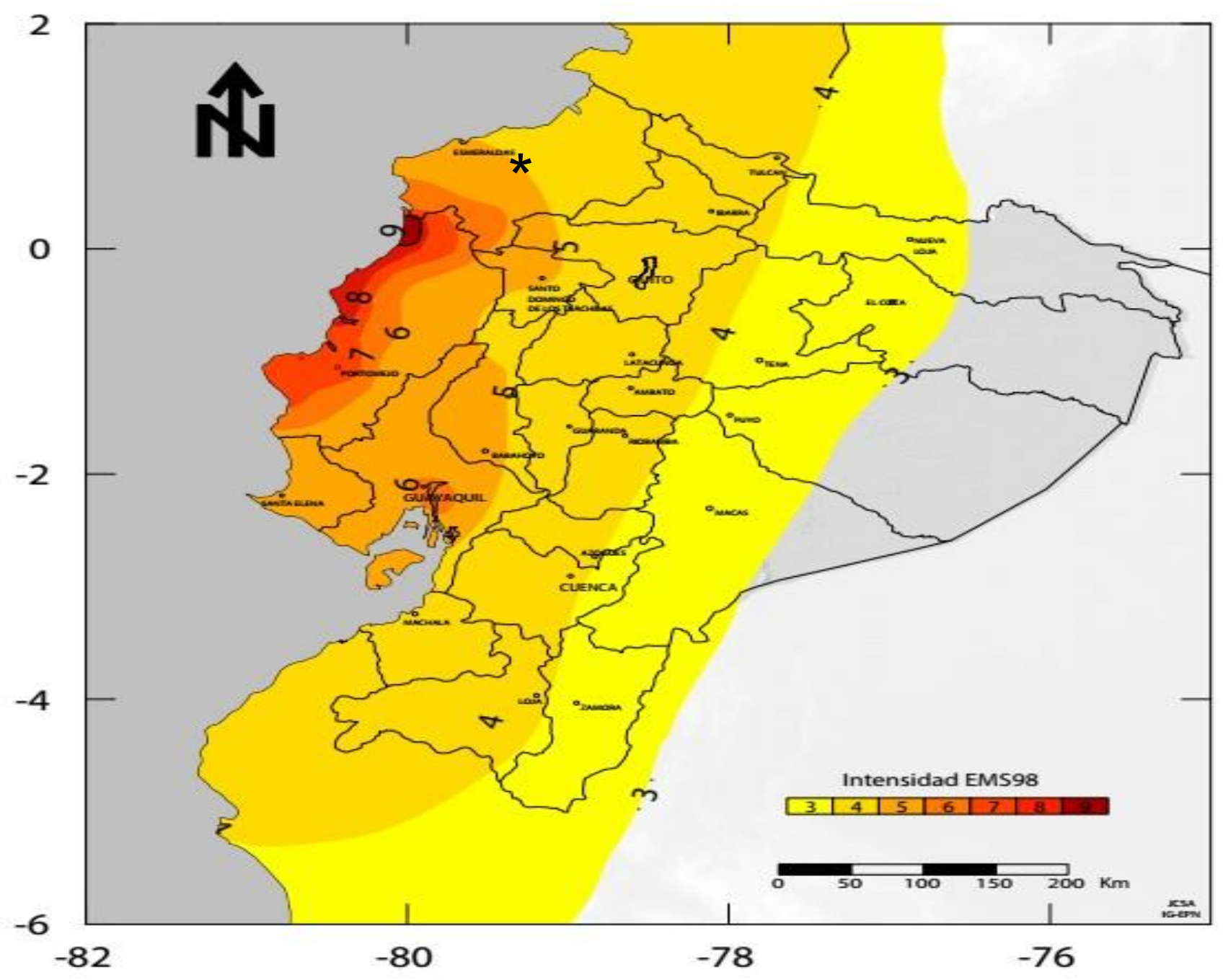

Figura 2: Ubicación del epicentro (*) e isosísmicas EMS-98 para el terremoto de Ecuador del 16 de abril de 2016. Fuente: Instituto Geofísico de la Escuela Politécnica Nacional (IGEPN).

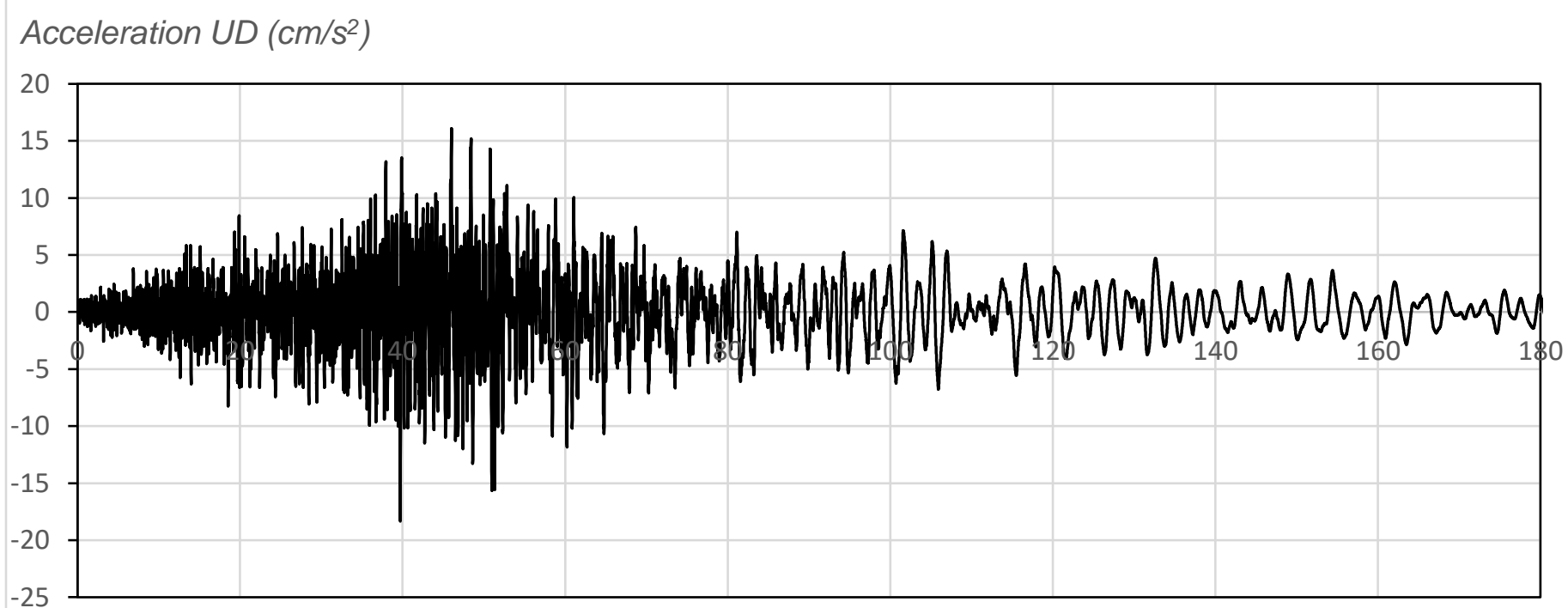

Time (s) 


\section{Acceleration NS $\left(\mathrm{cm} / \mathrm{s}^{2}\right)$}

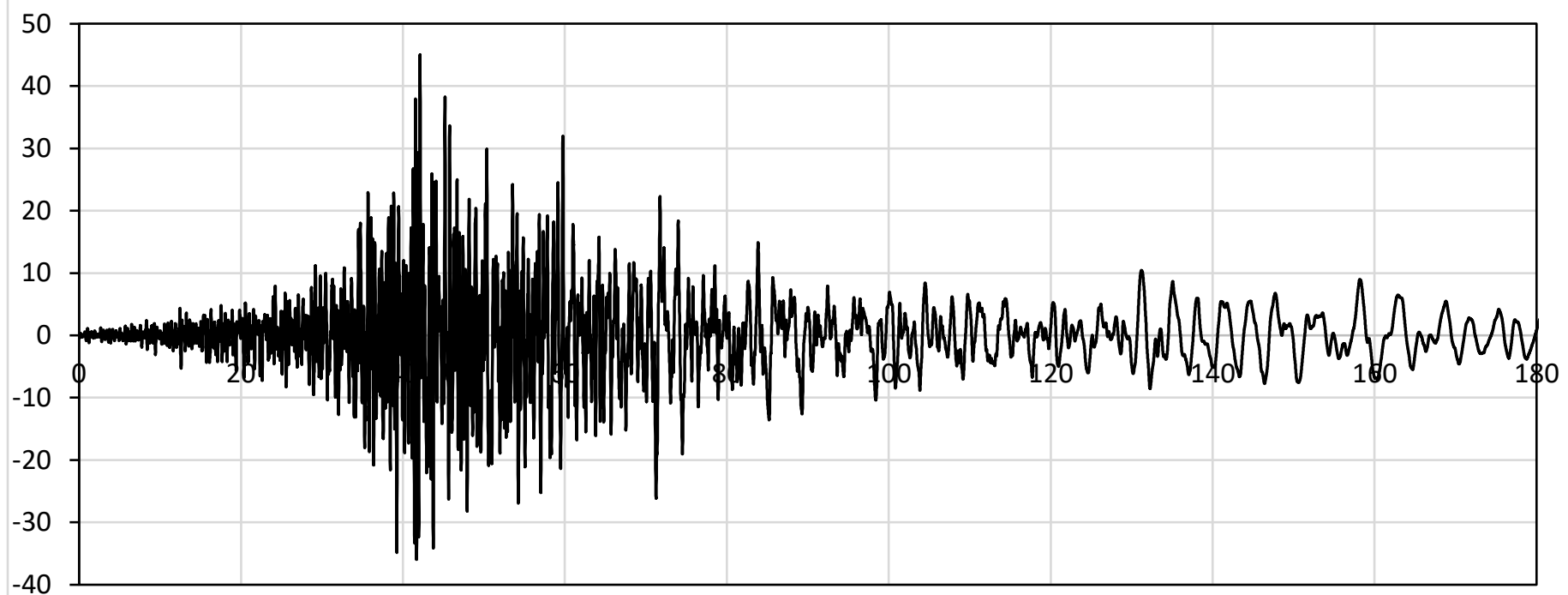

Time (s)

\section{Acceleration $\mathrm{EW}\left(\mathrm{cm} / \mathrm{s}^{2}\right)$}

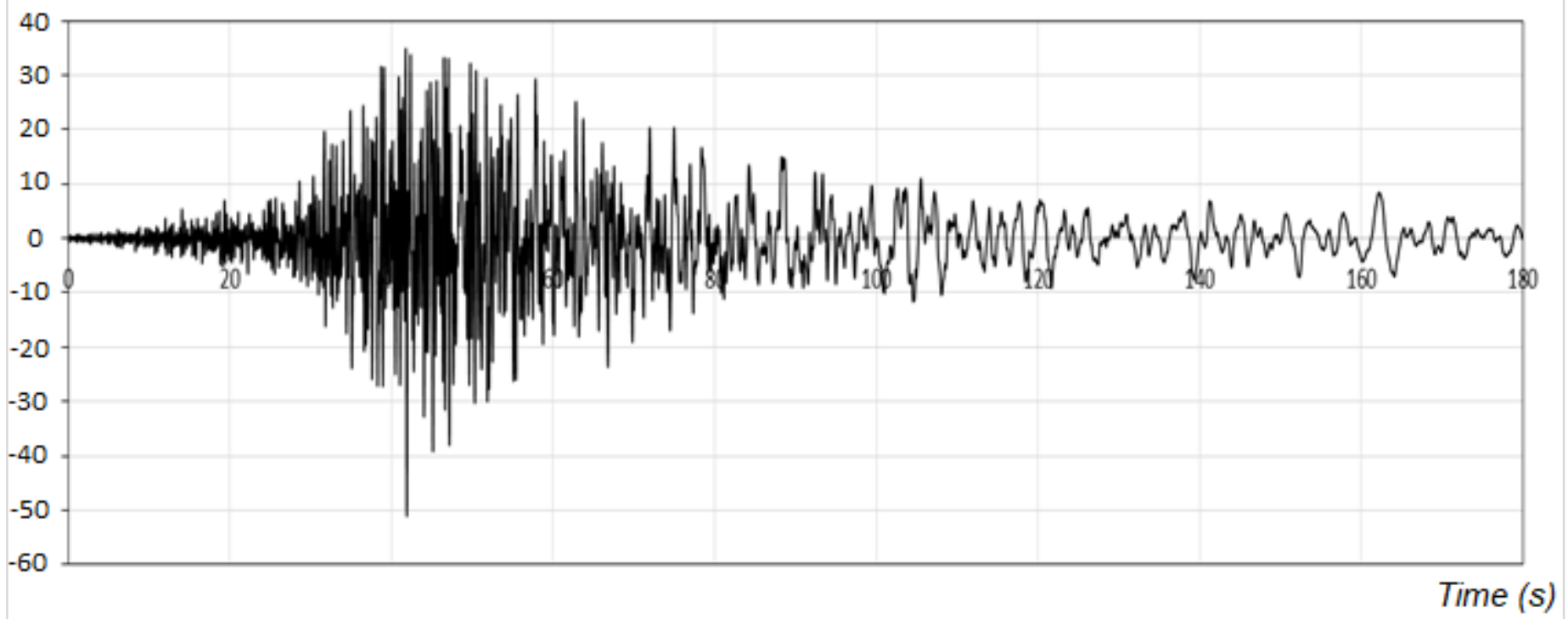

Figura 3: Aceleraciones sísmicas observadas en la estación AMIL. 


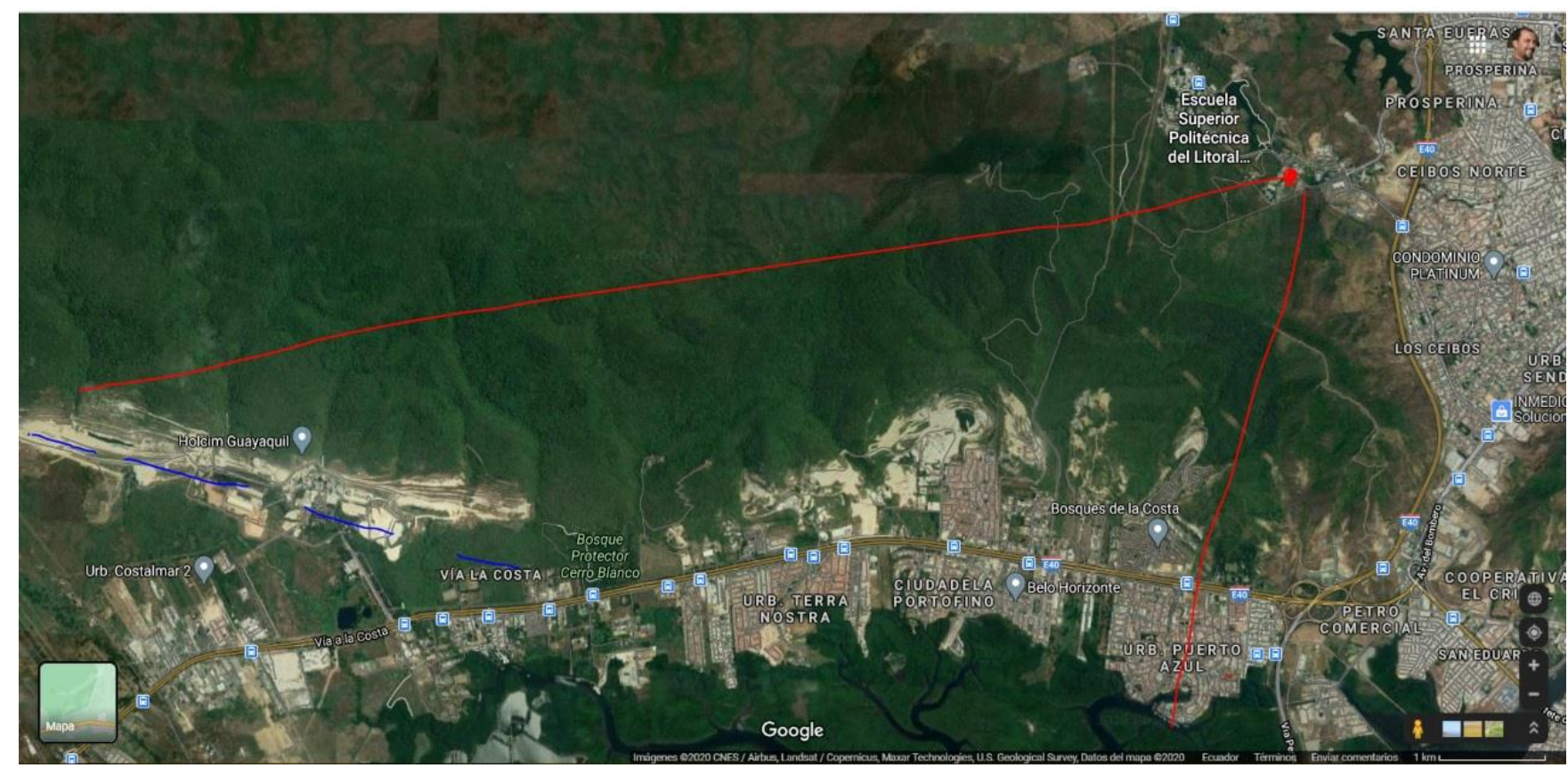

Figura 4: Campo de visión (líneas rojas) y ubicación (círculo rojo) de la cámara EPSOL. La falla de Chongón Colonche se ha marcado con una línea discontinua azul. La imagen fue tomada de Google Maps.

\section{Number of EQL occurrence}

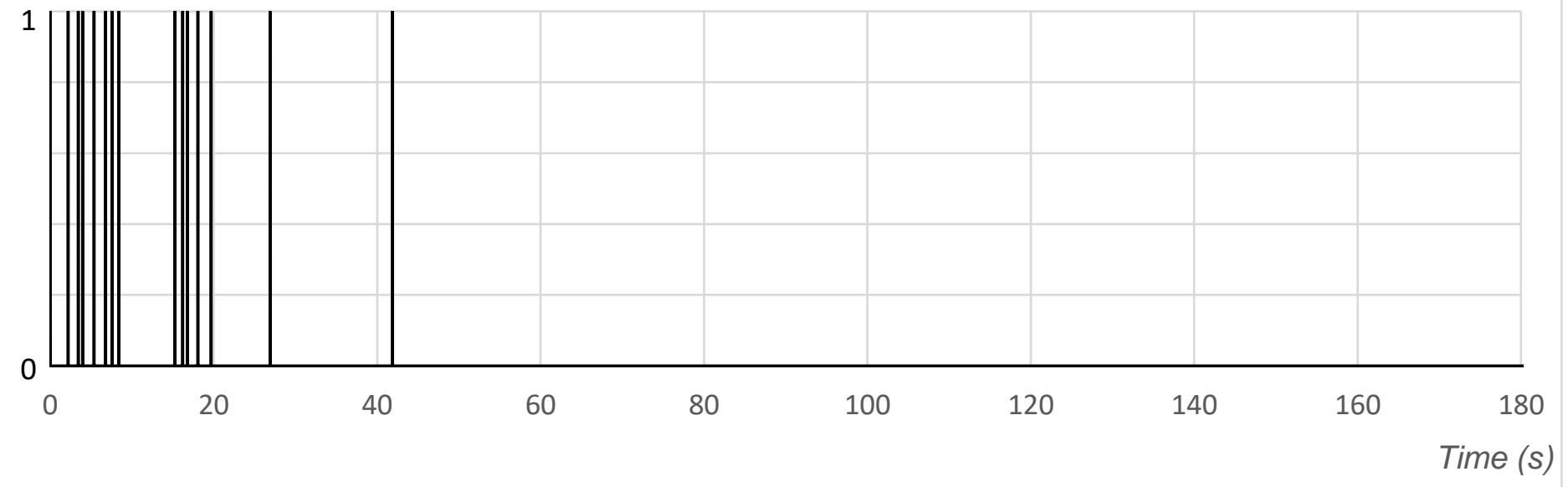

Figura 5: Diferencias de tiempo entre el primer EQL y los siguientes. Para la primera luz de terremoto se asignó arbitrariamente el instante cero segundos. Una línea vertical significa que ocurrió una luz sísmica.

\section{Metodología}

Para identificar una correlación temporal diferencial entre los EQL de Guayaquil y las aceleraciones sísmicas del suelo, solo es necesario superponer el acelerograma al diagrama de los EQL de la Fig. 5. De esta manera, se puede observar fácilmente si se produce la correlación temporal diferencial. Este método es particularmente útil cuando la cámara y el acelerómetro no están sincronizados. La distribución temporal de los EQL de Guayaquil coincide estrechamente con los valores extremos relativos de la aceleración de este a oeste. También podemos observar que la EQL más intensa registrada en la película (No. 6 de la Tabla 1) coincide con la mayor aceleración absoluta del acelerograma. Los resultados se muestran en la Fig.6. 


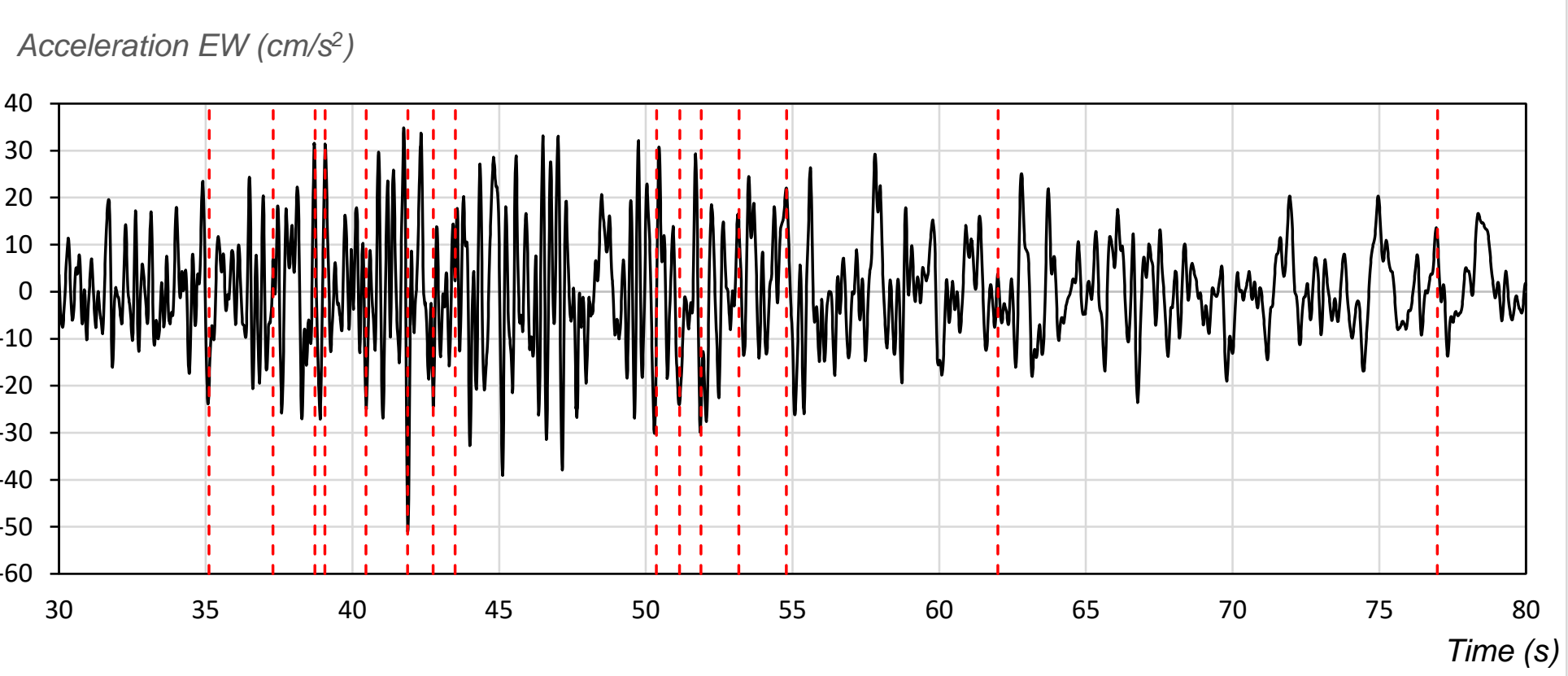

Figura 6: Correlación temporal diferencial entre los EQL (líneas rojas discontinuas) y los valores extremos relativos de la aceleración de este a oeste.

\section{Resultados}

En este trabajo se pudo demostrar una correlación temporal diferencial entre los EQL de Guayaquil y las aceleraciones máximas del suelo en la dirección este-oeste debido al terremoto. Una mirada a la grabación de la película en cámara lenta también nos permite darnos cuenta de que los EQL de Guayaquil se irradiaron en destellos esféricos desde la superficie de la Tierra hacia la atmósfera. Además, los EQL de Guayaquil coinciden morfológicamente con las luces sísmicas descritos por St-Laurent sobre los EQL de Saguenay de noviembre de 1988 a enero de 1989, así como con los destellos luminosos mencionados por Fidani sobre los EQL asociados con el terremoto de Aquila, Italia, del 6 de abril de 2009. Las imágenes de la grabación de la película sugieren que partículas cargadas se emitieron con simetría esférica desde la superficie de la Tierra a la atmósfera. Asimismo, la carga eléctrica en la superficie de la Tierra puede descargarse a la atmósfera si su campo eléctrico es lo suficientemente fuerte como para causar la ruptura dieléctrica del aire.

\section{Conclusiones}

Guayaquil está a unos $250 \mathrm{~km}$ del epicentro, lo que mostraría que los EQL no se limitan a las inmediaciones del epicentro. La correlación temporal diferencial que se muestra en este artículo sugeriría que las aceleraciones sísmicas del suelo registradas cerca de Guayaquil estaban vinculadas con los EQL de Guayaquil. Las aceleraciones sísmicas del suelo están relacionadas con las tensiones que actúan en la corteza terrestre subyacente. Si estas tensiones son lo suficientemente grandes, pueden fracturar rocas de la corteza terrestre. La fractura de la roca está asociada con la separación de la carga eléctrica, como se pudo demostrar en experimentos de laboratorio [2]. El mecanismo por el cual esta separación de carga en la corteza terrestre podría generar una carga eléctrica en la superficie de la Tierra fue tratada en varios artículos [3], [4], [5].

Además, podemos obtener información de los EQL, de las aceleraciones sísmicas del suelo y de su correlación temporal diferencial. Mirando de cerca la película, podemos darnos cuenta de que los EQL se irradian solo en ciertos puntos de la superficie de la Tierra a pesar de que las ondas sísmicas se propagan por toda la corteza terrestre, lo que nos lleva a sugerir que los procesos de generación de los EQL comienzan solo en ciertos puntos de la corteza terrestre. Los experimentos de laboratorio antes mencionados sobre la fracturación de rocas sugerirían que cada vez que se emite una luz de terremoto desde la superficie de la Tierra a la 
atmósfera, ha habido una fracturación de roca en la corteza terrestre subyacente.

Los terremotos de gran magnitud son eventos raros. Los EQL son eventos aún más raros. Sin embargo, ambos están vinculados. Los estudios relacionados con los EQL pueden ser la clave para anticipar grandes terremotos algún día.

\section{Agradecimientos}

Quisiera agradecer a M. Segovia, del IGEPN en Quito, por brindarme los datos sísmicos. También estoy muy agradecido con M. Mulas, de ESPOL en Guayaquil, por la valiosa información con relación a la cámara de seguridad. Igualmente deseo agradecer a mi hermano el profesor Juan Guillermo Lira Cacho, de la UNI en Lima, por haberme ayudado en muchas formas durante la preparación de este artículo.

\section{Referencias}

[1] C. Beauval et al., "Comparison of Observed Ground-Motion Attenuation for the 16 April
2016 Mw 7.8 Ecuador Megathrust Earthquake and Its Two Largest Aftershocks with Existing Ground-Motion Prediction Equations", Seismol. Res. Lett., vol. 88, pp. 287-299, Feb 2017.

[2] T. Bleier et al., "Correlation of pre-earthquake electromagnetic signals with laboratory and field rock experiments", Nat. Hazards Earth Syst. Sci.,vol. 10, pp. 1965-1975, Sep 2010.

[3] B. T. Brady and G. A. Rowell, "Laboratory investigation of the electrodynamics of rock fracture", Nature, vol. 321, pp. 488-492, May 1986.

[4] F. T. Freund, "Pre-earthquake signals - Part II: Flow of battery currents in the crust", Nat. Hazards Earth Syst. Sci., vol. 7, pp. 543-548, Sep 2007.

[5] R. Thériault et al., "Prevalence of Earthquake Lights Associated with Rift Environments", Seismol. Res. Lett., vol. 85, pp. 159-178, Jan 2014.

E-Mail: jlirac@unmsm.edu.pe 